\title{
Capacidade dos Profissionais de Saúde de Medicina e Enfermagem dos Cuidados de Saúde Primários em Identificar Doentes de Cuidados Paliativos
}

\section{Ability of Medical and Nursing Healthcare Professionals from Primary Care to Identify Palliative Care Patients}

Mariana van Innis', Manuel Luís Capelas², Alexandre Castro Caldas²

\begin{abstract}
RESUMO
INTRODUÇÃO: Os cuidados paliativos são cuidados de saúde especializados, dirigidos a indivíduos com doenças crónicas. De acordo com dados de estudos recentes, apenas uma parte da população portuguesa tem acesso a eles, sendo o número de indivíduos referenciados para equipas especializadas inferior ao esperado. Os cuidados de saúde primários podem desenvolver um papel importante na identificação e referenciação dos doentes, pois são o primeiro contacto da população com o Serviço Nacional de Saúde.
\end{abstract}

OBJETIVO: Avaliar a capacidade dos médicos e enfermeiros dos cuidados de saúde primários em identificar doentes de cuidados paliativos e determinar os fatores pessoais facilitadores.

MATERIAL E MÉTODOS: É um estudo analítico e transversal, efetuado através da aplicação de um questionário junto a 49 profissionais de saúde de medicina e enfermagem de dois Agrupamentos dos Centros de Saúde de Lisboa, entre junho e setembro de 2014. A análise estatística foi realizada através do programa SPSS ${ }^{\circledR} 20$.

RESULTADOS: Somente 14,3\% da amostra identificou corretamente os casos clínicos referenciáveis para cuidados paliativos. Dos vários fatores pessoais analisados, observámos uma relação estatisticamente significativa $(p<0,05)$ entre o contacto com a área dos cuidados paliativos na vida pessoal e a formação diferenciada e a identificação de doentes de cuidados paliativos por parte dos médicos e enfermeiros dos cuidados de saúde primários.

CONCLUSÕES: Os resultados obtidos sugerem a necessidade de reforçar a formação em cuidados paliativos junto dos profissionais de saúde e facilitar a comunicação entre os diferentes elementos envolvidos no processo de identificação e referenciação dos doentes.

PAlavRAS-Chave: Atitude do Pessoal de Saúde; Cuidados Paliativos; Cuidados de Saúde Primários; Médicos de Família; Pessoal de Enfermagem; Pessoal de Saúde; Portugal; Referenciação e Consulta

1. Unidade de Cuidados Paliativos, Hospital CUF Infante Santo, Lisboa, Portugal. 2. Centro de Investigação Interdisciplinar em Saúde, Instituto de Ciências da Saúde, Universidade Católica Portuguesa, Lisboa, Portugal.

Recebido: 10/08/2016 - Aceite: 19/04/2017 


\section{ABSTRACT}

INTRODUCTION: Palliative care is a specialized type of healthcare directed to individuals with chronic conditions. According to recent data, only a proportion of the Portuguese population has access to this care, the number of cases referred to specialized teams still falling below what would be expected. Doctors and nurses from primary care can play a relevant role in identifying and referring individuals, as they represent their first contact point with the Portuguese National Healthcare Service.

OBJECTIVE: Assess the ability of doctors and nurses from primary care to identify palliative care patients and determine facilitating personal factors.

MATERIAL \& METHODS: Analytical and cross-sectional study, undertaken by submitting a questionnaire to 49 medical and nursing healthcare professionals from two Primary Care Trusts in Lisbon, Portugal, between June and September 2014. Statistical analysis of the data was conducted using the SPSS ${ }^{\circledR} 20$.

RESULTS: Only 14.3\% of the sample correctly identified the clinical cases referred to palliative care. From the analyzed contributing factors, two were of statistical significance $(p<0.05)$ in identifying palliative care patients: personal involvement in this are and specific training.

CONCLUSIONS: The results obtained demonstrate the need it is essential to strengthen palliative care training among health professionals and facilitate communication between the different elements involved in identifying and referring patients.

KEYWORDS: Attitude of Health Personnel; Health Personnel; Nursing Staff; Palliative Care; Physicians, Family; Portugal; Primary Health Care; Referral and Consultation

\section{INTRODUÇÃO}

No âmbito da saúde, os cuidados paliativos modernos remontam da década de 60 do século XX, tendo como pioneira Cicely Saunders, em Inglaterra. Emergem em complemento ao modelo da medicina curativa, que encara a morte como uma "derrota", e realçam a necessidade crescente de oferecer cuidados rigorosos, científicos e de qualidade a indivíduos com doenças incuráveis e progressivas. Adicionalmente, constituem uma resposta ao envelhecimento da população, cada vez mais evidente à medida que a esperança média de vida à nascença aumenta e a natalidade diminui. ${ }^{1}$

Em 1990, a Organização Mundial de Saúde apresenta a primeira definição de cuidados paliativos, reformulada em 2002. Define, assim, os cuidados paliativos como "uma abordagem que visa melhorar a qualidade de vida dos doentes - e suas famílias - que enfrentam problemas decorrentes de uma doença incurável e/ou grave e com prognóstico limitado, através da prevenção e alívio do sofrimento, com recurso à identificação precoce e tratamento rigoroso dos problemas não só físicos, mas também dos psicossociais e espirituais". 2 São também cuidados interdisciplinares, que oferecem o conceito mais básico do cuidar: satisfazer as necessidades do indivíduo onde quer que ele seja cuidado, em casa ou numa instituição de saúde, de uma forma organizada e estruturada. $^{3}$
Os cuidados paliativos requerem, desta forma, uma referenciação e uma intervenção atempadas, de forma a garantir a qualidade dos cuidados de saúde prestados. Idealmente, devem ser instituídos desde o diagnóstico de risco de vida, de maneira a ser possível gerir adequadamente os sintomas, satisfazer as necessidades e estabelecer relações de confiança. ${ }^{1}$

Todavia, dados de estudos nacionais e internacionais apontam que este tipo de cuidados ainda é essencialmente dirigido a doentes oncológicos e/ou em fase terminal. Em Portugal, estima-se que, por ano, cerca de 60000 indivíduos necessitam de cuidados paliativos, mas apenas 6000 têm acesso a eles. ${ }^{4,5}$

Por outro lado, os cuidados de saúde primários representam geralmente o primeiro contacto da população com o Serviço Nacional de Saúde, pelo que os profissionais da área desempenham um papel primordial na identificação e na referenciação de doentes com necessidades paliativas para equipas diferenciadas.

Segundo a Organização Mundial de Saúde, os cuidados de saúde primários devem estar envolvidos nos cuidados paliativos, tornando-os mais acessíveis e globais à população. ${ }^{6}$ De facto, os médicos e os enfermeiros dos cuidados de saúde primários podem prestar assistência a doentes com necessidades paliativas, mas devem garantir a intervenção de equipas especializadas, em casos mais complexos. ${ }^{5}$ Embora partilhem princípios idênticos 
ao da medicina paliativa, como a abordagem holística, o envolvimento da família nos cuidados e a correta gestão dos tratamentos, evitando os que são fúteis, estes profissionais de saúde enfrentam vários obstáculos na prestação de cuidados paliativos, ${ }^{7-22}$ comprometendo, assim, a qualidade dos mesmos. Podemos realçar alguns, tais como:

- Escassa formação em cuidados paliativos, nomeadamente no controlo de certos sintomas e no domínio de alguns materiais, como as seringas infusoras;

- Dificuldade em abordar temas delicados, como o fim de vida;

- Incapacidade em identificar precocemente doentes de cuidados paliativos, sendo mais fácil realizar um prognóstico nas últimas semanas de vida ou nos doentes oncológicos. ${ }^{7-22}$

Estes obstáculos podem estar associados à formação de base dos médicos e dos enfermeiros, que se baseia principalmente nas vertentes preventiva e curativa das doenças, sendo pontual o ensino dos cuidados paliativos. Devido a estes obstáculos, alguns sintomas podem não estar devidamente controlados e o doente e a família podem ter falsas expectativas acerca da doença e do seu decurso, optando por tratamentos fúteis e agressivos, reduzindo, assim, a sua qualidade de vida. ${ }^{23,24}$

Para além destes obstáculos, também podemos destacar algumas causas que impedem ou atrasam o processo de referenciação para equipas especializadas de cuidados paliativos, nomeadamente:

- Conflitos entre os cuidados de saúde primários e os cuidados paliativos - os primeiros apontam razões como a falta de disponibilidade por parte dos peritos e a burocratização do Serviço de Saúde, para além de crerem que também são capazes de prestar cuidados de qualidade a este tipo de doentes;

- Incerteza do prognóstico;

- Inadequadas competências de comunicação;

- Pobres conhecimentos acerca dos cuidados paliativos e dos recursos disponíveis na comunidade;

- Escassez de suporte e de tempo;

- Recusa do doente e da família.,25

Por vezes, o doente e a família também podem constituir uma barreira, por não terem conhecimentos adequados sobre este tipo de cuidados e acreditarem que possa encurtar a sua esperança de vida. ${ }^{26}$

Perante estes factos, é pertinente determinarmos os fatores pessoais dos profissionais de saúde de medicina geral e familiar e de enfermagem de cuidados de saúde primários, que podem facilitar a identificação de doentes de cuidados paliativos, para uma possível referenciação.

\section{MATERIAL E MÉTODOS}

Para respondermos aos nossos objetivos, optámos por um estudo analítico e transversal.

Após aprovação do presente estudo pelo Conselho Científico da Universidade Católica Portuguesa e pela Comissão de Ética da Administração Regional de Saúde de Lisboa e Vale do Tejo, foram realizados pedidos de recolha de dados a três Agrupamentos de Centros de Saúde (ACES) da região de Lisboa, dos quais dois deram um parecer positivo.

A população-alvo foi, assim, composta por médicos e enfermeiros de dois ACES da área de Lisboa, sendo que foram excluídos os que exerciam funções em unidades ou equipas de cuidados paliativos.

Para podermos abranger um maior número de profissionais, optámos por uma amostra aleatória simples.

A colheita de dados foi realizada através da aplicação de um questionário junto aos profissionais de saúde, entre junho e setembro de 2014. Os questionários foram distribuídos pelos centros de saúde pertencentes aos ACES e preenchidos à mão por cada um dos participantes, não tendo havido contacto entre os mesmos e o investigador.

○úmero total da população-alvo foi fornecido pelas direções dos ACES em questão. Num total de 548 profissionais, 49 responderam ao questionário.

O questionário foi elaborado com a colaboração de três peritos de cuidados paliativos, ou seja, profissionais de saúde com formação avançada (mestrado ou doutoramento) e cuja atividade profissional se centra essencialmente na área. Posteriormente, foi testado por outros peritos de cuidados paliativos. Foi-Ihes pedido que avaliassem oito casos clínicos, determinando se eram ou não referenciáveis para cuidados paliativos. Dos oito, dois não obtiveram consenso nas respostas, pelo que foram excluídos do questionário final. Os casos clínicos foram construídos com base na literatura e com o apoio de três peritos da área. Os que foram considerados referenciáveis para cuidados paliativos compreendiam critérios de referenciação, internacionalmente aceites, dos quais se salientam os seguintes: sintomas múltiplos e intensos, com necessidade de intervenções complexas e utilização de recursos, evolução rápida, com crises frequentes, dilemas éticos, forte impacto emocional, antecedentes de toxicodependência e dificuldade de suporte familiar/ 
exaustão do cuidador informal. O questionário final foi dividido em duas partes. A primeira consistiu na caraterização pessoal e profissional dos participantes e a segunda na identificação dos casos clínicos referenciáveis para cuidados paliativos.

Ao longo da investigação, respeitámos os aspetos éticos e legais, como o anonimato, a confidencialidade e a proteção contra o prejuízo. Todos os participantes assinaram um consentimento informado e ficaram com um exemplar na sua posse, de forma a garantir que foram esclarecidos sobre os objetivos do estudo e os direitos que os assistem.

Os dados recolhidos foram tratados informaticamente através do programa SPSS (Statistical Package for the Social Sciences), versão 20.

Identificámos como variáveis independentes: género, idade, profissão, categoria profissional, tempo de exercício profissional (em geral e em cuidados de saúde primários), articulação profissional e pessoal com os cuidados paliativos e formação em cuidados paliativos, e, como variável dependente: capacidade dos médicos e enfermeiros dos cuidados de saúde primários em identificar doentes de cuidados paliativos.

Inicialmente, procedemos a uma análise descritiva e, posteriormente, a uma análise inferencial.

A análise descritiva pretendeu caraterizar a amostra em estudo, com base nas variáveis independentes e dependente. Para as variáveis numéricas, foram determinadas as seguintes medidas: a média, o mínimo e o máximo, a mediana e o desvio padrão. Para as variáveis categóricas, foram calculados os valores absolutos e as respetivas frequências.

Para verificarmos diferenças estaticamente significativas para as variáveis género, profissão e formação em cuidados paliativos, utilizámos o teste binomial.

Quanto à análise inferencial, pretendemos correlacionar cada variável independente com a dependente, sendo que esta última consistiu na capacidade dos profissionais de saúde de medicina e enfermagem em identificar doentes de cuidados paliativos. Para concretizarmos esta análise, criámos a variável dependente com base no número de acertos observados em cada caso clínico apresentado, em comparação com a resposta dos peritos. De notar que a variável género foi excluída, por ter uma disparidade significativa entre o número de participantes femininos e masculinos.

Ao avaliarmos as normalidades das diferentes distribuições, não identificámos as premissas necessárias para a realização de testes paramétricos, pelo que foram usa- dos testes não paramétricos, mais precisamente o de Sperman e o de Mann-Whitney. De referir que para a análise da correlação entre a variável tipo de formação com a variável dependente, empregámos o teste de ANOVA, após ter sido verificada a homogeneidade da amostra em questão. Considerámos $p<0,05$ como estatisticamente significativo.

\section{RESULTADOS}

Em termos de caraterização demográfica da amostra, constatamos que dos 49 participantes, 45 eram do género feminino e quatro do género masculino. $\bigcirc$ primeiro predominou em relação ao segundo. A média de idades rondou os 43,96 anos, sendo o desvio padrão de 9,95 anos.

Relativamente à caraterização profissional da amostra, dos 49 participantes, 32 eram enfermeiros e 17 médicos, sendo os primeiros predominantes em relação aos segundos. Em cada profissão, a categoria profissional mais representativa foram os enfermeiros $(28,6 \%)$ e os médicos, assistente e graduado (12,2\%) (Tabela 1).

TABELA 1. Categoria profissional da amostra $n=49$

\begin{tabular}{|l|c|c|}
\hline Grupo Profissional & $\mathrm{n}$ & $\%$ \\
\hline Enfermeiro & 14 & 28,6 \\
\hline Enf. Especialista & 7 & 14,3 \\
\hline Enf. Graduado & 11 & 22,4 \\
\hline Médico Interno & 3 & 6,1 \\
\hline Médico Assistente & 6 & 12,2 \\
\hline Médico Graduado & 6 & 12,2 \\
\hline Médico Sénior & 2 & 4,1 \\
\hline Total & 49 & 100 \\
\hline
\end{tabular}

Em relação ao tempo de exercício profissional, a média situou-se nos 19,55 anos, em geral, e nos 12,49 anos, na área dos cuidados de saúde primários, com um desvio padrão de 9,79 e 9,28 anos, respetivamente.

Quanto à caraterização relativa à articulação profissional e pessoal com os cuidados paliativos, dos 49 participantes, 28 articulavam com equipas ou unidades de cuidados paliativos, enquanto 21 não o faziam, e 23 afirmaram já ter tido contacto na sua vida pessoal com equipas ou unidades de cuidados paliativos, ao passo que 26 não.

Relativamente à caraterização quanto à formação em cuidados paliativos, 35 não tinham essa formação, enquanto 14 a tinham.

Em relação à caraterização sobre a capacidade em identificar doentes de cuidados paliativos, a média de respostas certas situou-se nos 4,2. O número mais represen- 
tativo de respostas certas correspondeu ao 4 (36,7\%). Constatamos que apenas 14,3\% da amostra conseguiu identificar corretamente todos os casos clínicos referenciáveis para cuidados paliativos (Tabela 2).

TABELA 2. Número de acertos da amostra $n=49$

\begin{tabular}{|c|c|c|}
\hline Número de Acertos & $\mathrm{n}$ & $\%$ \\
\hline 2 & 4 & 8,2 \\
\hline 3 & 6 & 12,2 \\
\hline 4 & 18 & 36,7 \\
\hline 5 & 14 & 28,6 \\
\hline 6 & 7 & 14,3 \\
\hline Total & 49 & 100 \\
\hline
\end{tabular}

Quanto à análise inferencial, das diferentes variáveis independentes analisadas, observamos uma relação estatisticamente significativa entre o contacto com a área dos cuidados paliativos na vida pessoal $[U=179,500, p=0,013$ $(p<0,05)]$ e a formação diferenciada $[U=153,500$, $p=0,035(p<0,05)]$ e a capacidade em identificar doentes de cuidados paliativos por parte da amostra.

\section{DISCUSSÃO}

Como acima descrito, existem vários obstáculos à identificação e referenciação de doentes de cuidados paliativos, por parte dos médicos e enfermeiros. No entanto, existem também fatores pessoais que podem influenciar este passo, como a idade, a formação de base, a experiência e o contacto com os cuidados paliativos.

Da análise dos dados que caraterizam a amostra em estudo, constatamos que o grupo feminino era predominante em relação ao masculino. Tal facto pode traduzir a "feminização da profissão médica". Atualmente, a percentagem de médicos do sexo feminino é superior à de médicos do sexo masculino, sobretudo nas faixas etárias inferiores, seguindo a tendência de outros países comparáveis. ${ }^{27}$ A profissão de enfermagem, por seu turno, também é essencialmente feminina, embora se constate nos dias de hoje um lento crescimento de enfermeiros do sexo masculino. ${ }^{27}$ Como antes referido, o fator género foi excluído da análise estatística, na medida em que existia uma diferença significativa entre o número de elementos femininos e masculinos, impossibilitando assim uma comparação equitativa. Relativamente à pesquisa bibliográfica quanto à influência deste fator na referenciação para cuidados paliativos, não existe um consenso, pois uma investigação sugere que ser do sexo feminino é estatisticamente significativo, enquanto um outro estudo revela que o género não tem relevância. ${ }^{28,29}$

Quanto à idade de ambos os grupos profissionais, que formam a amostra, a média estabeleceu-se nos 43,96 anos. Comparativamente ao panorama nacional, para os médicos, as faixas etárias com maior expressão são as que se situam abaixo dos 31 anos, com cerca de 18\%, e, para os enfermeiros, as dos 26-30 anos, com perto de $22 \%{ }^{30,31}$ Assim sendo, podemos admitir que a amostra está relativamente próxima das idades jovens, predominantes em ambos os grupos profissionais do setor de saúde português. Contudo, é importante salientar que, globalmente, a profissão médica é descrita como sendo uma profissão envelhecida, na medida em que mais de $54 \%$ dos seus membros tem mais de 50 anos. Todavia, parece haver uma "renovação geracional", pois o escalão até aos 39 anos já representa 31\% do total dos profissionais. ${ }^{32}$ A profissão de enfermagem, por sua vez, apresenta um ritmo de entrada de novos enfermeiros semelhante à reforma dos mesmos. ${ }^{33}$ De acordo com os dados obtidos, podemos afirmar que a idade não representa um fator estatisticamente significativo na capacidade dos profissionais de saúde em identificar doentes de cuidados paliativos. Contudo, há estudos que comprovam que a idade jovem dos profissionais de saúde, nomeadamente dos médicos de família, está associada a um maior número de doentes referenciados para cuidados paliativos, provavelmente graças à inclusão, mais visível nos últimos anos, do ensino e da prática desta área nas suas formações de base. ${ }^{34,35}$ Em contrapartida, os profissionais de saúde com uma idade mais avançada recorrem menos aos Cuidados Paliativos por terem mais experiência com a complexidade dos casos clínicos, nomeadamente em fim de vida, e uma visão mais cética quanto aos reais benefícios desta especialidade. ${ }^{35}$

Relativamente ao número total de médicos e enfermeiros portugueses, notamos que este último é superior ao primeiro. Segundo uma investigação realizada em Portugal, em 2010, a proporção enfermeiro/médico é de 1,2 enfermeiros por médico, exceto na área de saúde familiar, em que este número é quase equivalente. ${ }^{27}$ De facto, e de acordo com os dados fornecidos pelas respetivas direções dos ACES em estudo, a quantidade de médicos e enfermeiros é sensivelmente próxima: 259 médicos contra 289 enfermeiros. No entanto, a amostra era constituída maioritariamente por enfermeiros. Esta situação pode eventualmente ser justificada pela classe de enfermagem da área dos cuidados de saúde primários estar mais acessível e representar um "elo de ligação entre a família, os outros profissionais e os recursos da comunidade". ${ }^{36}$

Quanto à categoria profissional da amostra, os enfermeiros (generalistas) eram os mais representativos, seguidos dos médicos assistentes e graduados. Estes dados podem estar relacionados com a idade ainda jovem da amostra. De facto, a formação médica é de longa du- 
ração, sendo que a capacitação do profissional demora cerca de 9 anos para a especialidade de medicina geral e familiar (MGF). ${ }^{32}$ No caso dos enfermeiros, para além da idade jovem, também podemos apontar a baixa percentagem de enfermeiros especialistas, em Portugal: de acordo com a Ordem dos Enfermeiros, em 2013, dos 65872 profissionais, apenas 13083 tinham o título de especialista. ${ }^{27,31}$ Todavia, de acordo com um estudo realizado em 2013, o número de enfermeiros especialistas está a crescer, provavelmente graças a um maior investimento dos mesmos na sua formação académica. ${ }^{28}$ Segundo os dados obtidos, não existem diferenças estatisticamente significativas entre ambos os grupos profissionais (médicos e enfermeiros) na capacidade em identificar doentes de cuidados paliativos. Através da pesquisa bibliográfica, não foi possível encontrarmos investigações alusivas a este fator no domínio da referenciação para cuidados paliativos.

Em relação ao tempo de exercício profissional da amostra, em geral e na área dos cuidados de saúde primários, as médias foram 19,55 e 12,49 anos, respetivamente. Face a estes resultados, podemos constatar que uma parte da amostra trabalhava há menos tempo em cuidados de saúde primários do que em geral, tendo provavelmente exercido noutras áreas. De acordo com os dados, o tempo de exercício profissional, tanto em geral, como em cuidados de saúde primários, não é estatisticamente significativo na capacidade dos profissionais de saúde em identificar doentes de cuidados paliativos, corroborando outras investigações, em que os anos de experiência profissional também não constituíram um fator determinante na referenciação dos doentes para esta área. ${ }^{36}$ Contudo, um estudo demonstra que a prática no âmbito da saúde familiar ou medicina interna está relacionada com um maior número de doentes referenciados para cuidados paliativos, provavelmente pela proximidade existente entre o profissional de saúde e o núcleo doente/família, permitindo a abordagem de questões sensíveis, como o fim de vida. ${ }^{29}$

Relativamente à articulação profissional da amostra com a área dos cuidados paliativos, 28 elementos relacionavam-se, no âmbito do trabalho, com equipas ou unidades especializadas. Em contrapartida, 21 elementos não tinham essa relação profissional. De acordo com os dados obtidos, constatamos que não há uma diferença estatisticamente significativa entre ambos os grupos na capacidade de identificar doentes de cuidados paliativos. Todavia, a proximidade entre os cuidados de saúde primários e os cuidados paliativos pode facilitar a referenciação, na medida em que os profissionais de saúde ficam mais familiarizados com os recursos disponíveis na comunidade. ${ }^{37}$ De facto, como anteriormente foi descrito, um dos obstáculos na referenciação dos doentes para cuidados paliativos consiste no desconhecimento dos profissionais de saúde acerca dos apoios existentes no serviço de saúde e dos reais benefícios desta especialidade.

Quanto à articulação pessoal da amostra com os cuidados paliativos, 23 profissionais de saúde afirmaram ter tido um contacto prévio com equipas ou unidades especializadas, ao passo que 26 não o tiveram. Os dados obtidos demonstram que ambos os grupos têm uma diferença estatisticamente significativa na capacidade em identificar doentes de cuidados paliativos, sendo que o grupo de profissionais de saúde que já contactou, na sua vida pessoal, com equipas ou unidades especializadas, acertou mais do que o grupo que não teve esse contacto. De facto, uma investigação, realizada junto a um grupo de médicos, demonstrou que a experiência pessoal com a área de cuidados paliativos duplicou a referenciação, embora não fosse um fator estatisticamente significativo. ${ }^{37}$ Provavelmente, a relação pessoal com equipas ou unidades de cuidados paliativos permite um melhor conhecimento e consciencialização deste domínio. Vários estudos apontam igualmente que a comunicação e a articulação entre as diferentes especialidades resultam essencialmente de ligações pessoais e não de processos formais. ${ }^{38,39}$

Em relação aos conhecimentos da amostra, 14 profissionais de saúde tinham formação específica em cuidados paliativos, enquanto 35 não a tinham. Deste modo, podemos afirmar que era uma amostra predominantemente sem formação especializada. Mais ainda, verificamos que apenas dois elementos da amostra tinham formação avançada, isto é, mestrado. Os restantes com formação obtiveram-na através de ações soltas ou do curso básico. Embora tenha havido recentemente, em Portugal, um desenvolvimento na formação dos profissionais de saúde em cuidados paliativos, incluindo a mesma no currículo de base de vários cursos de medicina e enfermagem, a escassez de especialistas ainda constitui uma barreira na promoção deste tipo de cuidados. ${ }^{40}$ Os dados obtidos evidenciam que ambos os grupos têm uma diferença estatisticamente significativa na capacidade em identificar doentes de cuidados paliativos, sendo que o grupo de profissionais de saúde com formação diferenciada acertou mais do que o grupo sem essa formação. Efetivamente, a formação específica dos profissionais de saúde em cuidados paliativos facilita a referenciação, pois possibilita a clarificação de certos preconceitos e a criação de orientações: como identificar necessidades paliativas nos doentes, como prestar cuidados paliativos primários, quando recorrer a especialistas e como trabalhar de forma eficaz com estes últimos. ${ }^{41,42}$ Contudo, a educação por si só pode não ser suficiente, pelo 
que é fundamental valorizar a prática e a aprendizagem compartilhada, promovendo momentos de partilha de conhecimentos entre os profissionais dos cuidados de saúde primários e os especialistas na área dos cuidados paliativos. $^{38,43}$

Relativamente à diferenciação na capacidade em identificar doentes de cuidados paliativos entre os elementos com formação avançada e conhecimentos básicos, esta não é estaticamente significativa. No entanto, é importante salientar que os elementos com formação avançada representavam um número reduzido em relação aos demais, podendo assim enviesar este resultado.

Mais ainda, constatamos que apenas 14,3\% da amostra identificou corretamente os seis casos clínicos apresentados, podendo estes ser referenciáveis ou não para cuidados paliativos. Neste sentido, torna-se fundamental apostar nos fatores pessoais, que podem intervir neste processo, realçando o contacto e a formação em cuidados paliativos.

Podemos identificar várias limitações à presente investigação, sendo uma das principais o tamanho reduzido da amostra, em que o tempo de recolha de dados (quatro meses) não nos permitiu obter um maior número de participantes. Embora a amostra não seja representativa da população em estudo, possibilitou-nos analisar vários fatores e apontar novas linhas de investigação.

\section{CONCLUSÃO}

Na presente investigação, apenas 14,3\% da amostra conseguiu identificar corretamente os casos clínicos. Efetivamente, através da literatura, detetámos vários obstáculos na identificação e na referenciação dos doentes para equipas ou unidades de cuidados paliativos, por parte dos profissionais de saúde. Contudo, também existem fatores pessoais, inerentes ao médico e ao enfermeiro, que podem influenciar estes passos.

Dos vários fatores estudados, observámos uma relação estatisticamente significativa $(p<0,05)$ entre o contacto com a área dos cuidados paliativos na vida pessoal e a formação diferenciada e a identificação de doentes de cuidados paliativos por parte dos médicos e enfermeiros dos cuidados de saúde primários. Podemos, assim, afirmar que a "familiarização" dos profissionais de saúde com a área dos cuidados paliativos pode facilitar a identificação de doentes e a sua eventual referenciação para equipas ou unidades especializadas. Neste sentido, o estudo sugere a necessidade de promover a comunicação entre especialistas de diferentes áreas, nomeadamente entre os de cuidados de saúde primários e os de cuidados paliativos, e reforçar a formação em cuidados paliativos junto dos profissionais de saúde. Poderia ser interessante realizar o levantamento das necessidades de formação dos profissionais de saúde em cuidados paliativos, com o intuito de implementar e regulamentar a disciplina nos currículos de medicina e enfermagem.

NOTA: Este artigo é parte da Dissertação apresentada à Universidade Católica Portuguesa para obtenção do grau de Mestre em Cuidados Paliativos, sob a orientação do Prof. Doutor Alexandre Castro Caldas e coorientação do Prof. Doutor Manuel Luís Capelas.

CONFLITOS DE INTERESSE: Os autores declaram não ter qualquer conflito de interesse na realização do presente trabalho.

FONTES DE FINANCIAMENTO: Não existiram fontes externas de financiamento para a realização deste artigo.

CONFIDENCIALIDADE DOS DADOS: Os autores declaram ter seguido os protocolos da sua instituição acerca da publicação dos dados de doentes.

PROTEÇÃO DE PESSOAS E ANIMAIS: Os autores declaram que os procedimentos seguidos na elaboração do presente trabalho estão em conformidade com as normas das comissões de investigação clínica e de ética, bem como da declaração de Helsínquia e da Associação Médica Mundial.

CONFLICTS OF INTEREST: The authors declare that they have no conflicts of interest.

FINANCIAL SUPPORT: This work has not received any contribution, grant or scholarship.

CONFIDENTIALITY OF DATA: The authors declare that they have followed the protocols of their work center on the publication of data from patients.

PROTECTION OF HUMAN AND ANIMAL SUBJECTS: The authors declare that the procedures followed were in accordance with the regulations of the relevant clinical research ethics committee and with those of the Code of Ethics of the World Medical Association (Declaration of Helsinki).

\section{REFERÊNCIAS}

1. Barbosa A, Galriça Neto I. Manual de cuidados paliativos. $2^{\text {a }}$ ed. Lisboa: Faculdade de Medicina de Lisboa; 2010.

2. Davies E, Higginson IJ. The solid facts: palliative care. Copenhagen: World Health Organization; 2004.

3. EAPC: Definition of Palliative Care. European Association for Palliative Care; 2010 [acesso 2014 Set.]. Disponível em: http://www.eapcnet.eu/Corporate/AbouttheEAPC/DefinitionandAims.aspx.

4. Capelas ML. Cuidados paliativos: uma proposta para Portugal. Cad Saúde. 2009;2:51-57. 
5. Oliveira JE. Cuidar de doentes em fim de vida: a percepção dos médicos de família [dissertação]. Lisboa: Universidade de Lisboa, Faculdade de Medicina de Lisboa; 2010.

6. World Health Organization. Cancer Control - Knowledge into Action. WHO Guide for Effective Palliative Care. Geneva: WHO; 2007.

7. Munday D, Dale J, Barnett M. Out-of-hours palliative care in the UK: perspectives from general practice and specialist services. J R Soc Med. 2002;95:28-30.

8. Dumitrescu L, van den Heuvel W, van den Heuvel-Olaroui M. Experiences, knowledge and opinions on palliative care among Romanian general practitioners. Croatian Med J. 2003;47:142-7.

9. Barclay S, Todd C, Grande G, Lipscombe J. How common is medical training in palliative care? A postal survey of general practitioners. Br J Gen Pract. 2007;47:800-4.

10. Llyod-Williams M, Carter YH. General practice vocational training in the UK: what teaching is given in palliative care? Palliat Med. 2003;17:616-20.

11. Shipman C. How and why do GPs use specialist palliative care services? Palliat Med. 2002;16:241-6.

12. Aubin M, Vézina L, Allard P, Bergeron R, Lemieux AP. Les soins palliatifs: profil de pratique des médecins de la région de Québec. Can Fam Physician. 2001;47:1999-2005.

13. Taubert M, Nelson A. "Oh God, not a Palliative": out-of-hours general practitioners within the domain of palliative care. Palliat Med. 2010;24:501-9.

14. Shipman C, Addington-Hall J, Barclay S, Briggs J, Cox I, Daniels $\mathrm{L}$, et al. Educational opportunities in palliative care: what do general practitioners want? Palliat Med. 2001;15:191-6.

15. Mitchell G. Whither general practice palliative care? Aust Fam Physician. 2006;35:757.

16. Carvalho S, Botelho MA. Os cuidados paliativos no âmbito dos cuidados de saúde primários: as intervenções dos enfermeiros. Pensar Enferm. 2001;15:2-24.

17. Prem V, Karvannan H, Kumar SP, Karthikbabu S, Syed N, Sisodia $V$, et al. Study of nurses' knowledge about palliative care: a quantitative cross-sectional survey. Indian J Palliat Care. 2012;18:122-7.

18. Groot MM, Vernooij-Dassen MJ, Crul BJ, Grol RP. General practitioners (GPs) and palliative care: perceived tasks and barriers in daily practice. Palliat Med. 2005;19:111-8.

19. Mitchell GK. How well general practitioners deliver palliative care? A systematic review. Palliat Med. 2002;16:457-64.

20. Hanratty B. GP views on developments in palliative care services. Palliat Med. 2000;14:223-4.

21. Tan HM, O'Connor MM, Miles G, Klein B, Schattner P. GP and nurses' perceptions of how after-hours care for people receiving palliative care at home could be improved: a mixed methods study. BMC Palliat Care. 2009;8:13.

22. Ewing G, Rogers M, Barclay S, McCabe J, Martin A, Campbell $M$, et al. Palliative care in primary care: a study to determine whether patients and professionals agree on symptoms. Br J Gen Pract. 2006;56:27-34.

23. Beernaert K, Deliens L, De Vleminck A, Devroey D, Pardon $K$, Van den Block L, et al. Early identification of palliative care needs by family physicians: a qualitative study of barriers and facilitators from the perspective of family physicians, community nurses and patients. Palliat Med. 2014;28:480-90.

24. Weeks JC, Cook EF, O'Day SJ, Peterson LM, Wenger N, Reding D, et al. Relationship between cancer patients' predictions of prognosis and their treatment preferences. JAMA. 1998;279:1709-14.

25. Walshe C, Todd C, Caress AL, Chew-Graham C. Judgements about fellow professionals and the management of patients receiving palliative care in primary care: a quality study. $\mathrm{Br} J$ Gen Pract. 2008;58:264-72.
26. Devi P. A timely referral to palliative care team improves quality of life. Indian J Palliat Care. 2011;17(suppl.):S14-S6.

27. Dussault G, Fronteira I. Recursos humanos para a saúde (RHS): plano integrado no plano nacional de saúde 2011-2016 (Portugal). Researchgate; 2010 [acesso 2015 Jan.]. Disponível em: https://www.researchgate.net/publication/267851969 RECURSOS_HUMANOS_PARA_A_SAUDE_RHS_PLANO_INTEGRADO_NO_PLANO_NACIONAL_DE_SAUDE_2011-16 PORTUGAL.

28. Johnson C, Girgis A, Paul CL, Currow DC. Cancer specialists' palliative care referral practices and perceptions: results of a national survey. Palliat Med. 2008;22:51-7.

29. Kogan AC, Brumley R, Wilber K, Enguidanos S. Physician factors that influence patient referrals to end-of-life care. Am J Manag Care. 2012;18:416-22.

30. Ordem dos Médicos: Estatísticas Nacionais - Estatísticas por Distrito 2014. Ordem dos Médicos; 2014 [acesso 2015 Jan.]. Disponível em: www.ordemdosmedicos.pt.

31. Ordem dos enfermeiros: Dados estatísticos a 31-12-2013. Ordem dos Enfermeiros; 2014 [acesso 2015 Janeiro]. Disponível em: http://www.ordemenfermeiros.pt/membros/ DadosEstatisticos/2013/files/assets/common/downloads/ Dados\%20Estat.pdf.

32. Peixoto H, Loureiro A, Costa C, Nunes C, Duarte N. Estudo de Evolução Prospectiva de Médicos no Sistema Nacional de Saúde [Relatório Final]. Coimbra: Universidade de Coimbra; 2013.

33. Lobo MJ. Distribuição geográfica dos enfermeiros em Portugal [dissertação]. Bragança: Instituto Politécnico de Bragança; 2013.

34. Kogan AC, Brumley R, Wilber K, Enguidanos S. Physician factors that influence patient referrals to end-of-life care. Am J Manag Care. 2012;18:416-22.

35. Keating NL, Landrum MB, Rogers SO, Baum SK, Virnig BA, Huskamp HA, et al. Physician factors associated with discussions about end-of-life care. Cancer. 2010;116:998-1006.

36. Ramos V, Seixas T, Pardal P, Pereira ML, Barbosa M, Marquês L, et al. Papel do Enfermeiro de Saúde Familiar nos CSP: pressupostos para a sua implementação. Lisboa: Ministério da Saúde, Grupo Técnico para o Desenvolvimento dos Cuidados de Saúde Primários; 2012.

37. Ahluwalia SG, Fried TR. Physician factors associated with outpatient palliative care referral. Palliat Med. 2009;23:608-15.

38. Gardiner C, Gott M, Ingleton C. Factors supporting good partnership working between generalist and specialist palliative care services: a systematic review. $\mathrm{Br} J$ Gen Pract. 2012;62:353-62.

39. Oishi A, Murtagh FE. The challenges of uncertainty and interprofessional collaboration in palliative care for non-cancer patients in the community: a systematic review of views from patients, carers and health-care professionals. Palliat Med. 2014;28:1081-98.

40. Centeno C, Clark D, Rocafort J, Flores LA, Greenwood A, Brasch S, et al. Atlas of palliative care in Europe. London: European Association for Palliative Care; 2013.

41. Kavalieratos D, Mitchell EM, Carey TS, Dev S, Biddle AK, Reeve BB, et al. "Not the "grim reaper service": an assessment of provider knowledge, attitudes, and perceptions regarding palliative care referral barriers in heart failure. J Am Heart Assoc. 2014;3:1-11.

42. Walshe C, Chew-Graham C, Todd C, Caress A. What influences referrals within community palliative care services? A qualitative case study. Social Sci Med. 2008;67:137-46.

43. Pype P, Symons L, Wens J, Van den Eynden B, Stes A, Deveugele M. Health care professionals' perceptions towards lifelong learning in palliative care for general practitioners: a focus group study. BMC Fam Pract. 2014;15:36. 